\title{
REAVALIAÇÃO A LONGO PRAZO DO TRATAMENTO CIRÚRGICO DA SÍNDROME DO TÚNEL DO CARPO POR INCISÃO PALMAR E UTILIZAÇÃO DO INSTRUMENTO DE PAINE ${ }^{\circledR}$
}

\author{
CARPAL TUNNEL SYNDROME: REASSESSMENT OF LONG-TERM OUTCOMES WITH THE USE OF THE PAINE ${ }^{\circledR}$ \\ RETINACULATOME DURING SURGERY THROUGH A PALMAR INCISION.
}

\author{
Luciana Leonel dos Santos ${ }^{1}$, Marco Aurélio Branco ${ }^{1}$, Lia Mitamoto Meirelles², João Baptista Gomes dos Santos ${ }^{3}$, \\ flávio Faloppa ${ }^{4}$, Walter Manna Albertoni ${ }^{5}$, Carlos Henrique Fernandes ${ }^{6}$
}

\begin{abstract}
RESUMO
A síndrome do túnel do carpo (STC) é a síndrome compressiva mais comum e a cirurgia de liberação do retináculo dos flexores uma das mais realizadas no mundo. Desde a via aberta clássica, mini-incisões até a endoscópica, o sucesso obtido com a cirurgia a curto prazo está bem estabelecido, porém os estudos a longo prazo ainda são escassos e avaliam, principalmente, sinais clínicos e sintomas. O objetivo deste estudo é avaliar os pacientes tratados pela incisão palmar e utilização do instrumento de Paine ${ }^{\circledR}$ com no mínimo seis anos de pós-operatório (98 meses em média). Foram feitas avaliações pré e pós-operatórias da força de preensão palmar, polpa-polpa, lateral e tridigital utilizando um dinamômetro de pressão. A sensibilidade dos dedos foi mensurada por meio de monofilamentos de nylon. Foi observado que com o passar do tempo houve melhora da sensibilidade e a força se manteve inalterada.
\end{abstract}

Descritores: Síndrome do túnel carpal; Força da mão; Sensação; Resultado de tratamento.

\section{SUMMARY}

Carpal tunnel syndrome (CTS) is the most commonly diagnosed and treated entrapment neuropathy. Surgical treatment involving the clearance of the flexor retinaculum is widely employed. Open, minimally-invasive and endoscopic surgical techniques have all been described as treatment options for CTS and short-term success with these methods is well established. Long-term studies, however, are scarce and usually evaluate only clinical signs and symptoms. The objective of this study is to evaluate patients treated by a palmar incision and by the use of the Paine retinaculatome (6 years post-op minimum; mean is 98 months). We assessed palmar, pulp to pulp, lateral and tridigital prehension strength using a dynamometer. Finger sensitivity was measured using nylon monofilaments. We observed that while sensitivity improved with time, grip and prehension strength remained unchanged.

Keywords: Carpal tunnel syndrome; Hand strength; Sensation; Treatment outcome.

\section{INTRODUÇÃO}

A compressão nervosa mais comum é a do nervo mediano que ocorre na área em que este atravessa a região do carpo, caracterizando a síndrome do túnel do carpo (STC). Esta pode ocorrer por aumento do volume das estruturas contidas nele ou pela diminuição do espaço no interior do túnel. Segundo estudos anatômicos a região mais estreita do túnel é no hâmulo do hamato e a flexão do punho provoca a compressão do nervo pela margem proximal do retináculo dos flexores.

O túnel do carpo é a região por onde passam os tendões flexores dos dedos e o nervo mediano em direção aos dedos da mão. Seu assoalho é formado pelo arco côncavo dos ossos cárpicos cobertos pelos seus ligamentos. O teto do túnel é formado pelo retináculo dos flexores, uma banda fibrosa imediatamente acima do nervo mediano, que tem sua inserção do lado radial, no tubérculo do osso escafóide e no osso trapézio e do lado ulnal, no osso pisiforme e no hâmulo do hamato. No túnel o nervo localiza-se em posição volar aos tendões flexores superficiais dos dedos médio e anular. A cirurgia para liberação do túnel do carpo é um assunto corrente na literatura, motivo de diversas publicações, principalmente a partir dos anos 50. Algumas relatam vários tipos de complicações ${ }^{(1,2,3,4)}$ e muitas outras relatam excelentes resultados e baixos índices de complicações ${ }^{(1,3,5,6)}$.

Nos últimos anos há uma crescente utilização dos métodos endoscópicos para a liberação do túnel do carpo, com o objetivo de agilizar o retorno dos pacientes ao trabalho e diminuir a morbidade ${ }^{(7)}$. A desvantagem dessa técnica é o elevado número de complicações operatórias e o alto custo do instrumental e do treinamento do cirurgião ${ }^{(1,3,8,9)}$.

Este trabalho tem por objetivo reavaliar os resultados a longo prazo do tratamento cirúrgico em 112 pacientes portadores de síndrome do túnel do carpo, operados por incisão palmar, utilizando o instrumento de Paine ${ }^{\circledR}$.

Trabalho realizado na Disciplina de Cirurgia da Mão e Membro Superior do Departamento de Ortopedia e Traumatologia da Universidade Federal de São Paulo - DOT/UNIFESP

Endereço para correspondência: Rua Borges Lagoa no 783, $5^{\circ}$ andar - CEP 04038-032 - Vila Clementino - São Paulo - SP - E mail: luci.leo@bol.com.br

1. Médico Residente do Departamento de Ortopedia e Traumatologia

2. Fisioterapeuta e Especialista em Terapia da mão

3. Doutor em Ciências, Chefe de Clínica da Disciplina de Cirurgia da Mão e Membro Superior do Departamento de Ortopedia e Traumatologia

4. Doutor em Ciências, Chefe de Clínica da Disciplina de Cirurgia da

5. Professor Titular do Departamento de Ortopedia e Traumatologia. Pró-reitor de Extensão

6. Doutor em Ciências, Médico da Disciplina de Cirurgia da Mão e Membro Superior do Departamento de Ortopedia e Traumatologia

Trabalho recebido em: 02/06/05 aprovado em 19/08/05 


\section{MATERIAL E MÉTODO}

De outubro a dezembro de 2004 reavaliamos os pacientes operados entre março de 1995 e março de 1998, na "Casa da Mão" da Disciplina de Çirurgia da Mão e Membro Superior - Departamento de Ortopedia e Traumatologia /UNIFESP. Foram 89 pacientes, totalizando 112 mãos, submetidos à liberação cirúrgica do túnel do carpo, por incisão palmar e instrumento de Paine ${ }^{\circledR(10)}$. Conseguimos localizar e examinar 45 pacientes, sendo que o restante dos pacientes não respondeu à solicitação de comparecimento e um paciente foi excluído por ter sofrido acidente vascular cerebral com conseqüente perda de força da mão, o que alteraria a avaliação pós-operatória. No total tivemos 44 pacientes, com 56 mãos reavaliadas.

Entre os 44 pacientes avaliados, 13 aparecem duas vezes em nossa casuística por terem sido operados de ambos os lados $(11$ e 29, 13 e 16, 14 e 15,17 e 22, 18 e 27, 19 e 25, 24 e 53, 28 e 45, 30 e 31, 37 e 40, 38 e 39, 41 e 43, 48 e 50). As mãos operadas são listadas por ordem cronológica do dia da cirurgia e nenhuma mão foi submetida a mais de um procedimento cirúrgico.

O tempo de reavaliação pósoperatória variou de 80 meses a 117 meses, com uma média de 98 meses.

$A$ idade, na época da reavaliação, variou de 38 a 77 anos, com uma média de 57 anos. Na época da cirurgia a idade variou de 18 a 79 anos, com média de 46 anos.

Quanto ao sexo, 2 (4,5\%) eram do sexo masculino e 42 (95,5\%) do feminino.

Quanto à mão acometida, 36 $(81,8 \%)$ pacientes tinham queixas bilaterais, $8(18,2 \%)$ tinham na mão direita e $0(0,0 \%)$ na esquerda. A mão dominante era a direita em $40(89,5 \%)$ pacientes, a esquerda em $1(1,7 \%)$ e $3(8,8 \%)$ eram ambidestros. A cirurgia foi realizada na mão direita em 25 (56,8\%) pacientes, na mão esquerda em $6(13,6 \%)$ e $13(29,6 \%)$ bilateral.

$\mathrm{Na}$ Tabela 1 estão contidos os dados referentes aos pacientes segundo o número de ordem, iniciais do nome, e avaliação da força de preensão palmar, polpa-polpa, lateral, tridigital e na Tabela 2 a avaliação da sensibilidade por meio de monofilamentos do $1^{\circ}, 2^{\circ}, 3^{\circ}, 4^{\circ}$ e $5^{\circ}$ dedos, pré e pós-operatória. Avaliação pré-operatória da força de preensão palmar, pol-

\begin{tabular}{|c|c|c|c|c|c|c|c|c|}
\hline \multirow[t]{2}{*}{$\begin{array}{c}\mathrm{N} .{ }^{\circ} \\
\text { Ordem }\end{array}$} & \multicolumn{4}{|c|}{$\begin{array}{c}\text { Força (Kgf) } \\
\text { Pré-operatório }\end{array}$} & \multicolumn{4}{|c|}{$\begin{array}{c}\text { Força (Kgf) } \\
\text { Pós-operatório tardio }\end{array}$} \\
\hline & $\mathrm{P}$ & P-P & P-L & P-T & $\mathrm{P}$ & P-P & P-L & P-T \\
\hline 1 & & & & & 27 & 5,0 & 7,0 & 5,5 \\
\hline 2 & & & & & 20 & 4,0 & 6,0 & 5,5 \\
\hline 3 & & & & & 24 & 3,5 & 5,0 & 3,5 \\
\hline 4 & & & & & 12 & 3,5 & 6,0 & 3,0 \\
\hline 5 & & & & & 30 & 3,0 & 6,0 & 3,5 \\
\hline 6 & & & & & 30 & 5,0 & 7,5 & 6,0 \\
\hline 7 & & & & & 26 & 5,0 & 3,5 & 5,5 \\
\hline 8 & & & & & 24 & 2,5 & 4,5 & 3,0 \\
\hline 9 & & & & & 32 & 7,5 & 8,5 & 7,5 \\
\hline 10 & & & & & 24 & 5,5 & 8,0 & 7,0 \\
\hline 11 & & & & & 28 & 5,0 & 5,5 & 4,0 \\
\hline 12 & & & & & 24 & 5,0 & 6,5 & 5,5 \\
\hline 13 & & & & & 32 & 6,5 & 8,0 & 6,5 \\
\hline 14 & 28 & 4,5 & 6,5 & 6 & 22 & 4,5 & 7,5 & 6,5 \\
\hline 15 & 26 & 4 & 4 & 4,5 & 26 & 4,0 & 6,0 & 5,0 \\
\hline 16 & 28 & 2,5 & 3 & 3,5 & 30 & 5,5 & 7,5 & 6,5 \\
\hline 17 & 28 & 2,5 & 7 & 5 & 32 & 5,0 & 7,5 & 6,0 \\
\hline 18 & 22 & 5,5 & 6 & 4 & 24 & 4,5 & 7,0 & 5,0 \\
\hline 19 & 26 & 3 & 3,5 & 4 & 30 & 4,0 & 5,5 & 3,5 \\
\hline 20 & 30 & 2,5 & 5 & 4,5 & 26 & 2,5 & 3,5 & 3,0 \\
\hline 21 & 30 & 6,5 & 7,5 & 7 & 30 & 6,0 & 6,5 & 3,5 \\
\hline 22 & 30 & 4,5 & 7,5 & 7 & 28 & 5,0 & 7,0 & 5,0 \\
\hline 23 & 38 & 6 & 6,5 & 6,5 & 34 & 6,0 & 7,5 & 7,0 \\
\hline 24 & 28 & 4,5 & 6,5 & 5,5 & 23 & 4,5 & 6,5 & 5,5 \\
\hline 25 & 30 & 5 & 4 & 5,5 & 32 & 7,5 & 6,0 & 6,0 \\
\hline 26 & 32 & 5,5 & 7 & 8 & 26 & 5,0 & 7,0 & 6,5 \\
\hline 27 & 28 & 6 & 6 & 5,5 & 30 & 6,0 & 7,5 & 5,5 \\
\hline 28 & 30 & 5 & 5 & 5 & 32 & 4,5 & 6,0 & 5,5 \\
\hline 29 & 20 & 5 & 6 & 6,5 & 22 & 4,5 & 5,5 & 4,5 \\
\hline 30 & 20 & 5 & 5 & 5,5 & 20 & 5,5 & 8,0 & 6,5 \\
\hline 31 & 22 & 3,5 & 4,5 & 7,5 & 24 & 5,5 & 8,0 & 6,5 \\
\hline 32 & 30 & 7 & 10 & 7 & 28 & 5,0 & 9,5 & 6,5 \\
\hline 33 & 30 & 7 & 8 & 7 & 26 & 6,0 & 7,0 & 6,0 \\
\hline 34 & 22 & 4,5 & 7 & 5,5 & 28 & 5,0 & 7,0 & 6,0 \\
\hline 35 & 28 & 6 & 8 & 6 & 34 & 8,5 & 8,5 & 8,0 \\
\hline 36 & 30 & 4 & 3,5 & 5 & 32 & 4,0 & 6,0 & 4,5 \\
\hline 37 & 28 & 4,5 & 6,5 & 6,5 & 34 & 6,0 & 6,5 & 6,5 \\
\hline 38 & 38 & 6 & 8,5 & 8,5 & 34 & 3,5 & 8,0 & 8,0 \\
\hline 39 & 34 & 5 & 8 & 6,5 & 32 & 4,5 & 7,5 & 6,0 \\
\hline 40 & 28 & 5,5 & 5,5 & 6,5 & 30 & 7,0 & 4,5 & 7,0 \\
\hline 41 & 22 & 4 & 6 & 3,5 & 26 & 4,0 & 2,0 & 2,5 \\
\hline 42 & 22 & 5 & 6 & 5,5 & 30 & 7,0 & 7,5 & 6,5 \\
\hline 43 & 22 & 4,5 & 4,5 & 6,5 & 24 & 4,0 & 2,0 & 2,5 \\
\hline 44 & 16 & 4 & 3,5 & 4 & 30 & 4,0 & 5,0 & 4,0 \\
\hline 45 & 28 & 5,5 & 5,5 & 5,5 & 34 & 7,0 & 6,0 & 6,5 \\
\hline 46 & 22 & 5,5 & 6 & 4,5 & 24 & 4,0 & 5,5 & 5,5 \\
\hline 47 & 30 & 5 & 5,5 & 5,5 & 24 & 6,5 & 8,0 & 7,5 \\
\hline 48 & 16 & 4,5 & 5,5 & 5 & 20 & 4,5 & 6,5 & 5,5 \\
\hline 49 & 32 & 5,5 & 7,5 & 6 & 40 & 6,0 & 8,5 & 7,5 \\
\hline 50 & 16 & 3,5 & 5,5 & 4,5 & 16 & 3,0 & 6,0 & 5,0 \\
\hline 51 & 16 & 3,5 & 4 & 4 & 24 & 3,0 & 6,5 & 4,0 \\
\hline 52 & 26 & 3 & 4,5 & 5 & 26 & 4,0 & 4,5 & 5,0 \\
\hline 53 & 22 & 3,5 & 5,5 & 4 & 20 & 3,5 & 6,0 & 3,0 \\
\hline 54 & 34 & 5 & 7 & 7 & 42 & 7,5 & 8,5 & 8,0 \\
\hline 55 & 32 & 5,5 & 5 & 6 & 42 & 7,5 & 8,5 & 8,0 \\
\hline 56 & 20 & 3,5 & 3,5 & 3,5 & 20 & 2,0 & 4,0 & 3,5 \\
\hline
\end{tabular}

Tabela 1 - Dados referentes a 56 mãos operadas de 44 pacientes com Síndrome do Túnel do Carpo segundo o número de ordem, iniciais do nome, força de preensão palmar $(P)$, polpa-polpa $(P-P)$, lateral $(P-L)$ e tridigital $(P-T)$, no pré-operatório e pós-operatório. pa-polpa, lateral e tridigital foi realizada em $43(76,8 \%)$ mãos. Na reavaliação o teste foi aplicado a 56 mãos.

Avaliação pré-operatória da sensibilidade dos dedos da mão foi feita por meio de monofilamentos de nylon em 44 (78,6\%) mãos. Na reavaliação o teste foi aplicado a 56 mãos.

Para a mensuração da força de preensão palmar, polpa-polpa, lateral e tridigital foi utilizado um dinamômetro de preensão palmar hidráulico, ajustado na segunda posição e um dinamômetro de preensão digital hidráulico, ambos Baseline (Irvington, N.Y., U.S.A.). Para a avaliação foram efetuadas três mensurações em com o máximo de força possível, e anotada a média em quilograma-força. Os indivíduos estavam sentados, com o braço aduzido paralelo ao tronco, cotovelo fletido em 90 graus, antebraço e punho em posição neutra. A sensibilidade era pesquisada por meio do teste de monofilamentos de nylon (Estesiômetro ${ }^{\circledR}$ ), fabricado por SORRI, Bauru, Brasil. O conjunto consiste de um jogo de sete tubos, cada um contendo um par de monofilamentos. O número de cada fio indica com precisão a força axial necessária para envergar os filamentos que são de cores diferentes e correspondem à graduação em gramas. Verde - 0,05 g; azul - 0,20 g; violeta - 2,00 g; vermelho escuro - 4,00 g; laranja - 10,00 g; vermelho magenta - 300,00 g.

Para agilizar a avaliação, eram pesquisadas apenas as polpas digitais dos dedos das mãos. $\mathrm{O}$ exame era demonstrado em uma área de pele com sensibilidade normal e solicitavase ao paciente mexer o dedo quando sentisse o toque, era realizado sem observação visual do paciente, de modo que o monofilamento de nylon ficasse perpendicular à superfície da pele da polpa digital. A força exercida era suficiente para curvar o monofilamento. O teste era iniciado com o monofilamento mais leve, 0,05 g (verde). Caso não houvesse resposta do paciente, prosseguia-se o exame com o filamento imediatamente mais pesado (0,20 $\mathrm{g}$ - azul), e assim progressivamente. Os filamentos de 0,05g e 0,20 g (verde e azul) eram aplicados até três vezes em cada local, sendo suficiente apenas uma única resposta positiva para confirmar a sensibilidade. Os outros eram testados apenas uma vez. Em todas as cirurgias foi utilizado o instrumento cirúrgico 
desenvolvido por Paine, confeccionado em aço inoxidável, com uma haste achatada no plano frontal e uma placa de base no plano horizontal, formando entre si um ângulo de $135^{\circ}$

\section{MÉTODO ESTATÍSTICO}

Para avaliar a evolução das mãos em relação ao período pré-operatório e o período pós-operatório para as variáveis força de preensão palmar, polpa-polpa, lateral e tridigital, usamos o teste de Wilcoxon, pois havia interesse em verificar a evolução ao longo do tempo.

Este mesmo teste foi utilizado para a variável sensibilidade nos dedos, onde o tempo foi fixado em pré e pós-operatório para verificar como se comportava cada dedo ao longo do tempo. O nível de significância fixado em todos os casos foi sempre igual ou menor do que 0,05 (5\%).

Quando a estatística calculada apresentou um valor significante usamos um asterisco $\left(^{*}\right)$ para caracterizá-la. Caso contrário, isto é, não significante, usamos NS.

As médias foram calculadas e apresentadas apenas a título de informação.

\section{TERMINOLOGIA ANATOMICA}

A "Terminologia Anatômica" utilizada foi a da Sociedade Brasileira de Anatomia em Terminologia Anatômica, 2001.

\section{RESULTADOS}

A Tabela 1 apresenta as mensurações de força de preensão palmar, polpa-polpa, lateral e tridigital das 43 mãos que tiveram avaliações pré e pós-operatórias. A análise estatística é apresentada na Tabela 3 e ilustrada no Gráfico 1 segundo a força de preensão palmar, polpa-polpa, lateral e tridigital, respectivamente.

A Tabela 2 apresenta os resultados da sensibilidade dos dedos de 44 mãos que tiveram avaliações pré e pós-operatórias. A análise estatística é apresentada na Tabela 4 e o Gráfico 2 ilustra a sensibilidade em suas variáveis no pré e pós-operatório.

\section{DISCUSSÃO}

A síndrome do túnel do carpo é a síndrome compressiva mais comum, e a divisão do retináculo dos flexores é a cirurgia mais realizada no membro superior, em todo o mundo.

São poucos os estudos na literatura em que é realizado um seguimento a longo prazo de pacientes tratados cirurgicamente da Síndrome do túnel do carpo. O tempo de seguimento pós-operatório é variado nos diversos trabalhos, chegando até 72 meses de

\begin{tabular}{|c|c|c|c|c|c|c|c|c|c|c|}
\hline \multirow{2}{*}{$\begin{array}{l}N^{\circ} \text { de } \\
\text { Ordem }\end{array}$} & \multicolumn{5}{|c|}{$\begin{array}{c}\text { Sensibilidade Pré-Operatório } \\
\text { (g) }\end{array}$} & \multicolumn{5}{|c|}{$\begin{array}{l}\text { Sensibilidade Pós- } \\
\text { operatório tardio (g) }\end{array}$} \\
\hline & $1^{\circ}$ & $2^{\circ}$ & $3^{\circ}$ & $4^{\circ}$ & $5^{\circ}$ & $1^{\circ}$ & $2^{\circ}$ & $3^{\circ}$ & $4^{\circ}$ & $5^{\circ}$ \\
\hline 1 & & & & & & 0,05 & 0,05 & 0,05 & 0,05 & 0,05 \\
\hline 2 & & & & & & 2,00 & 0,20 & 0,20 & 0,20 & 0,20 \\
\hline 3 & & & & & & 0,20 & 2,00 & 0,05 & 0,05 & 2,00 \\
\hline 4 & & & & & & 0,05 & 0,05 & 0,05 & 0,05 & 0,05 \\
\hline 5 & & & & & & 0,20 & 0,05 & 0,05 & 0,05 & 0,05 \\
\hline 6 & & & & & & 0,05 & 0,05 & 0,05 & 0,05 & 0,05 \\
\hline 7 & & & & & & 0,05 & 0,05 & 0,05 & 0,05 & 0,05 \\
\hline 8 & & & & & & 0,05 & 0,05 & 0,05 & 0,05 & 0,05 \\
\hline 9 & & & & & & 0,05 & 0,05 & 0,05 & 0,05 & 0,05 \\
\hline 10 & & & & & & 0,05 & 0,05 & 0,05 & 0,05 & 0,05 \\
\hline 11 & & & & & & 2,00 & 2,00 & 2,00 & 2,00 & 2,00 \\
\hline 12 & & & & & & 0,05 & 0,05 & 0,05 & 0,05 & 0,05 \\
\hline 13 & 0,05 & 0,05 & 0,05 & 0,05 & 0,05 & 0,05 & 0,05 & 0,05 & 0,05 & 0,05 \\
\hline 14 & 0,05 & 0,05 & 0,05 & 0,05 & 0,05 & 0,05 & 0,05 & 0,05 & 0,05 & 0,05 \\
\hline 15 & 0,05 & 0,05 & 0,05 & 0,05 & 0,05 & 0,05 & 0,05 & 0,05 & 0,05 & 0,05 \\
\hline 16 & 0,20 & 0,05 & 0,05 & 0,05 & 0,05 & 0,05 & 0,05 & 0,05 & 0,05 & 0,05 \\
\hline 17 & 0,05 & 0,05 & 0,05 & 0,05 & 0,05 & 0,05 & 0,05 & 0,05 & 0,20 & 0,20 \\
\hline 18 & 0,05 & 0,05 & 0,05 & 0,05 & 0,05 & 0,05 & 0,05 & 0,05 & 0,05 & 0,05 \\
\hline 19 & 0,20 & 0,20 & 0,05 & 0,20 & 0,20 & 0,05 & 0,05 & 0,05 & 0,05 & 0,05 \\
\hline 20 & 10,00 & 10,00 & 10,00 & 0,20 & 0,20 & 0,20 & 0,20 & 0,20 & 0,05 & 0,05 \\
\hline 21 & 0,05 & 0,05 & 0,05 & 0,20 & 0,05 & 0,20 & 0,05 & 0,05 & 0,05 & 0,05 \\
\hline 22 & 0,20 & 0,20 & 0,20 & 0,20 & 0,20 & 2,00 & 0,05 & 0,05 & 0,20 & 0,20 \\
\hline 23 & 0,20 & 0,20 & 0,20 & 0,20 & 0,20 & 0,05 & 0,05 & 0,05 & 0,05 & 0,05 \\
\hline 24 & 0,20 & 0,20 & 0,20 & 0,20 & 0,20 & 0,05 & 0,05 & 0,05 & 0,05 & 0,05 \\
\hline 25 & 0,20 & 0,20 & 0,20 & 0,05 & 0,05 & 0,05 & 0,20 & 0,20 & 0,05 & 0,05 \\
\hline 26 & 0,20 & 0,20 & 0,20 & 0,20 & 0,20 & 2,00 & 0,05 & 0,20 & 0,20 & 0,20 \\
\hline 27 & 0,05 & 0,05 & 0,05 & 0,05 & 0,05 & 0,05 & 0,05 & 0,05 & 0,05 & 0,05 \\
\hline 28 & 2,00 & 2,00 & 2,00 & 2,00 & 2,00 & 0,05 & 0,05 & 0,05 & 0,05 & 0,05 \\
\hline 29 & 0,20 & 0,20 & 0,20 & 0,20 & 0,20 & 2,00 & 2,00 & 2,00 & 2,00 & 0,20 \\
\hline 30 & 2,00 & 2,00 & 2,00 & 2,00 & 2,00 & 0,05 & 0,05 & 0,05 & 0,05 & 0,05 \\
\hline 31 & 2,00 & 2,00 & 2,00 & 2,00 & 2,00 & 0,05 & 0,05 & 0,05 & 0,05 & 0,05 \\
\hline 32 & 0,20 & 0,20 & 0,20 & 0,20 & 0,20 & 0,20 & 0,20 & 0,05 & 0,05 & 0,05 \\
\hline 33 & 0,20 & 0,20 & 0,05 & 0,20 & 0,05 & 0,05 & 0,05 & 0,05 & 0,05 & 0,05 \\
\hline 34 & 0,20 & 0,20 & 0,20 & 0,20 & 0,20 & 0,05 & 0,20 & 0,20 & 0,20 & 0,20 \\
\hline 35 & 0,20 & 0,20 & 0,20 & 0,20 & 0,20 & 0,05 & 0,05 & 0,05 & 0,05 & 0,05 \\
\hline 36 & 2,00 & 2,00 & 2,00 & 2,00 & 0,20 & 0,20 & 0,20 & 0,20 & 0,20 & 0,05 \\
\hline 37 & 0,20 & 0,20 & 0,20 & 0,20 & 0,20 & 0,05 & 0,05 & 0,05 & 0,05 & 0,05 \\
\hline 38 & 0,20 & 0,20 & 0,20 & 0,20 & 0,20 & 0,20 & 0,20 & 0,05 & 0,05 & 0,05 \\
\hline 39 & 0,20 & 0,05 & 0,20 & 0,05 & 0,05 & 0,05 & 0,05 & 0,05 & 0,05 & 0,05 \\
\hline 40 & 0,20 & 0,20 & 0,20 & 0,20 & 0,20 & 0,05 & 0,05 & 0,05 & 0,05 & 0,05 \\
\hline 41 & 10,00 & 4,00 & 4,00 & 0,20 & 0,20 & 4,00 & - & - & 0,05 & 0,05 \\
\hline 42 & 0,20 & 0,20 & 0,20 & 0,20 & 0,20 & 2,00 & 2,00 & 0,20 & 0,20 & 0,20 \\
\hline 43 & 4,00 & 10,00 & 10,00 & 0,05 & 0,05 & 2,00 & - & - & 0,05 & 0,20 \\
\hline 44 & 0,20 & 0,20 & 0,20 & 0,20 & 0,20 & 0,05 & 0,05 & 0,05 & 0,05 & 0,05 \\
\hline 45 & 0,20 & 0,20 & 0,20 & 0,20 & 0,20 & 2,00 & 0,05 & 0,05 & 0,05 & 0,20 \\
\hline 46 & 0,20 & 0,20 & 0,20 & 0,20 & 0,20 & 0,05 & 0,05 & 0,05 & 0,05 & 0,05 \\
\hline 47 & 0,20 & 0,20 & 0,20 & 0,20 & 0,20 & 2,00 & 2,00 & 2,00 & 0,20 & 0,20 \\
\hline 48 & 4,00 & 4,00 & 4,00 & 0,20 & 0,05 & 0,05 & 0,05 & 0,05 & 0,05 & 0,05 \\
\hline 49 & 0,20 & 0,20 & 0,20 & 0,20 & 0,05 & 0,05 & 0,05 & 0,05 & 0,05 & 0,05 \\
\hline 50 & 0,20 & 0,20 & 0,20 & 0,20 & 0,20 & 0,05 & 0,05 & 0,05 & 0,05 & 0,05 \\
\hline 51 & 0,20 & 0,20 & 0,20 & 0,20 & 0,20 & 0,20 & 0,20 & 0,20 & 0,20 & 0,20 \\
\hline 52 & 2,00 & 2,00 & 2,00 & 0,20 & 0,05 & 0,20 & 0,20 & 0,05 & 0,05 & 0,05 \\
\hline 53 & 0,05 & 0,05 & 0,05 & 0,05 & 0,05 & 0,05 & 0,05 & 0,20 & 0,05 & 0,05 \\
\hline 54 & 0,20 & 0,20 & 0,20 & 0,20 & 0,20 & 0,20 & 0,20 & 0,20 & 0,20 & 0,20 \\
\hline 55 & 4,00 & 0,20 & 0,20 & 0,20 & 0,20 & 0,05 & 0,05 & 0,05 & 0,05 & 0,05 \\
\hline 56 & 4,00 & 4,00 & 4,00 & 2,00 & 2,00 & 0,20 & 0,20 & 0,20 & 0,20 & 0,20 \\
\hline
\end{tabular}

Tabela 2 - Dados referentes a 56 mãos operadas de 44 pacientes com Síndrome do Túnel do Carpo segundo o número de ordem, iniciais do nome e sensibilidade do 10 ao 50 dedos no pré-operatório e pós-operatório tardio. seguimento ${ }^{(1,11,12)}$. Não foram encontrados estudos de seguimento a longo prazo no tratamento da STC utilizando, exclusivamente, o instrumento de Paine ${ }^{\circledR}$. Neste estudo, o seguimento médio dos pacientes foi 8 anos e 2 meses. A STC ocorre normalmente entre a quarta e sexta década(6), dado concordante com nossa casuística, na época da cirurgia, em que $80 \%$ dos pacientes pertencem a esta faixa etária.

Observamos uma incidência no sexo feminino de $95,5 \%$, fatos concordantes com a literatura, em que a grande maioria dos trabalhos registra uma maior incidência da STC no sexo feminino ${ }^{(12,13)}$.

O acometimento bilateral é normalmente o mais encontrado nos diversos trabalhos, seguido pelo acometimento da mão direita e depois da mão esquerda isoladamente ${ }^{(14)}$, coincidente à nossa casuística em que encontramos $81,8 \%$ de bilateralidade e nenhuma isoladamente na mão esquerda. O método de avaliação da sensibilidade da mão usando monofilamentos foi bem estabelecido para avaliar os resultados após liberação cirúrgica do túnel do carpo ${ }^{(15,16)}$. Essa avaliação é fundamental para confirmar a integridade das estruturas nervosas e as mudanças na sensibilidade da mão após a cirurgia.

Outro método útil para avaliar a função da mão é a mensuração da força, feita com dinamômetros e podem ser medidas as forças de preensão palmar, polpa-polpa, lateral e tridigital ${ }^{(17,18,19)}$. A comparação com mão contralateral não deve ser usada como parâmetro por haver uma alta incidência de bilateralidade(20).

Nos pacientes submetidos à liberação do túnel do carpo, a força da mão retorna à força máxima préoperatória após seis meses, sendo que $15 \%$ a $20 \%$ dos pacientes nunca voltam a ter sua força original, devido à alteração na configuração dos ossos do carpo ou à perda do efeito de polia do retináculo(21). Naqueles em que há perda, esta é em torno de $20 \%$. Alguns autores relatam perda de força tardia, após 10 meses a dois anos, nos pacientes operados ${ }^{(12)}$. Quando avaliada a recuperação da força, usando a via de acesso clássica, observou que em relação ao pré-operatório a força de preensão e a força polpapolpa tiveram retorno ao mesmo valor de força pré-operatória entre o $3^{\circ}$ e $6^{\circ}$ mês ${ }^{(20)}$. Quando utilizado a via endoscópica, observou o retorno da força de preensão palmar e a força polpa-polpa ao redor da $6^{a}$ semana e $3^{a}$ semana, respectiva- 


\begin{tabular}{l|c|c|c}
\hline \hline $\begin{array}{c}\text { Força de } \\
\text { preensão }\end{array}$ & Pré-operatório & Pós-operatório & Análise estatistica \\
\hline palmar & 26,51 & 28,16 & zcalc $=-2,035^{*} \quad \mathrm{p}=0,042$ \\
\hline polpa-polpa & 4,68 & 5,04 & zcalc $=-1,706$ NS $p=0,088$ \\
\hline lateral & 5,82 & 6,5 & zcalc $=-2,742^{*} p=0,006$ \\
\hline tridigital & 5,57 & 5,59 & zcalc $=-0,453$ NS $p=0,651$ \\
\hline \hline
\end{tabular}

Tabela 3 - Análise estatística das médias da força de preensão palmar, polpa-polpa, lateral e tridigital no pré e pós-operatório de liberação do túnel do carpo

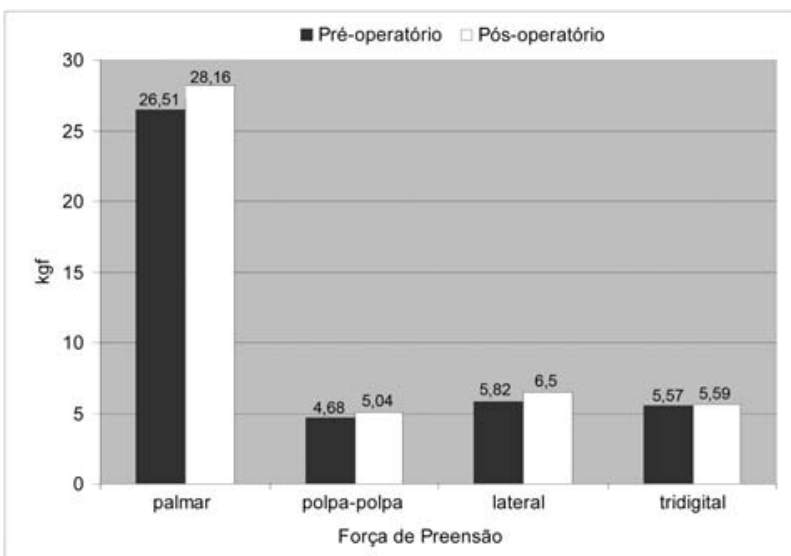

Grafico 1 - Média da força de preensão palmar, polpa-polpa, lateral e tridigital no pré e pós-operatório de liberação do túnel do carpo

mente ${ }^{(22)}$. Comparando-se a incisão clássica com a dupla incisão encontrou-se retorno da força mais precoce na dupla incisão(23) A cirurgia aberta padrão para liberação do túnel do carpo é o método preferido de tratamento porque continua sendo tão efetiva quanto as outras alternativas, sendo tecnicamente mais fácil e apresenta menor risco de complicações e custos adicionais (1,2) $^{(1 .}$

Neste estudo, as médias de força de preensão palmar encontradas foram 26,51 kgf no pré-operatório e 28,16 kgf a longo prazo, sendo que houve diferença estatisticamente significante. As médias de força de preensão polpa-polpa encontradas foram 4,68 kgf no pré-operatório e 5,04 kgf a longo prazo, sendo que não houve diferença estatisticamente significante. As médias de força de preensão lateral encontradas foram 5,82 kgf no pré-operatório e $6,50 \mathrm{kgf}$ a longo prazo, sendo que houve diferença estatisticamente significante. As médias de força de preensão tridigital encontradas foram 5,57 kgf no pré-operatório e 5,59 kgf a longo prazo, sendo que não houve diferença estatisticamente significante. Podemos

\begin{tabular}{c|c|c|c}
\hline \hline & Pré-operatório & Pós-operatório & Análise estatística \\
\hline $1^{\circ}$ dedo & 0,83 & 0,48 & zcalc $=-2,704^{\star} \mathrm{p}=0,007$ \\
\hline $2^{\circ}$ dedo & 0,79 & 0,23 & zcalc $=-3,161^{\star} \mathrm{p}=0,002$ \\
\hline $3^{\circ}$ dedo & 0,82 & 0,18 & zcalc $=-3,424^{\star} \mathrm{p}=0,001$ \\
\hline $4^{\circ}$ dedo & 0,4 & 0,13 & zcalc $=-3,951^{\star} \mathrm{p}=0,000$ \\
\hline $5^{\circ}$ dedo & 0,34 & 0,09 & zcalc $=-3,620^{\star} \mathrm{p}=0,000$ \\
\hline \hline
\end{tabular}

Tabela 4 - Análise estatística das médias da sensibilidade no pré e pós-operatório de liberação do túnel do carpo

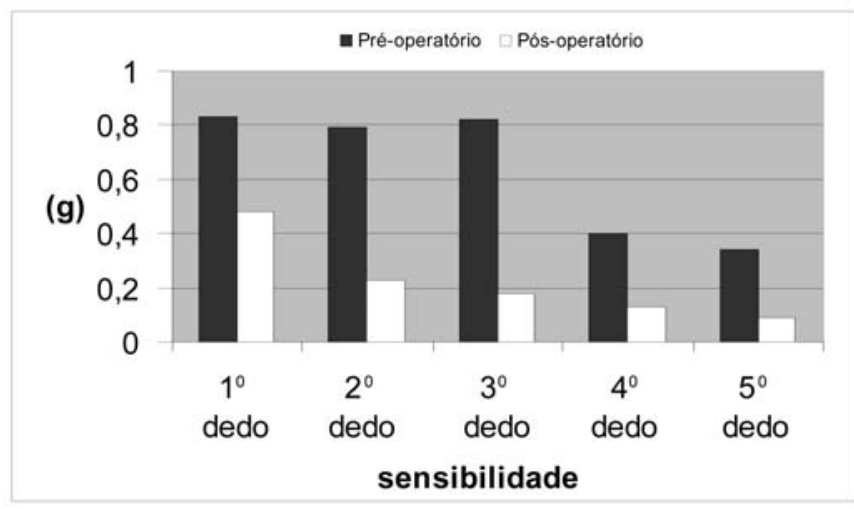

Gráfico 2 - Média da sensibilidade no pré e pós-operatório de liberação do túnel do carpo

sugerir que a longo prazo os pacientes tratados cirurgicamente por esta técnica apresentaram uma melhora global das forças a longo prazo.

A melhora da sensibilidade no pós-operatório varia nos diversos estudos de 17 a $65 \%{ }^{(16,20)}$. Na comparação entre um grupo submetido à liberação endoscópica e outro à cirurgia clássica, não observaram diferenças estatisticamente significantes entre eles e entre as mensurações pré e pós-operatórias ${ }^{(24)}$. No grupo de mãos estudado nesta pesquisa, todas apresentaram, na análise estatística, diferenças favoráveis e significantes entre o pré e os seguimentos pós-operatórios, nos cinco dedos.

\section{CONCLUSÃO}

Observamos que ao longo do tempo, a sensibilidade dos dedos apresentou melhora em relação ao pré-operatório.

A força muscular manteve-se semelhante ao pré-operatório, após longo tempo de seguimento pós-operatório.

\section{REFERÊNCIAS BIBLIOGRÁFICAS}

1. Gerritsen AA, Uitdehaag BM, van Geldere D, Scholten RJ, de Vet HC, Bouter LM. Systematic review of randomized clinical trials of surgical treatment for carpal tunnel syndrome. Br J Surg 2001; 88:1285-95.

2. Scholten RJ, Gerritsen AA, Uitdehaag BM., van Geldere D, de Vet HC, Bouter LM. Surgical treatment options for carpal tunnel syndrome. Cochrane Database Syst Rev: CD003905, 2004

3. Thoma A, Veltri K, Haines T, Duku E. A systematic review of reviews comparing the effectiveness of endoscopic and open carpal tunnel decompression. Plast Reconstr Surg 2004; 113:1184-91.

4. Zumiotti AV, Ohno PE, Prada FS, Azze RJ. Complicações do tratamento cirúrgico da síndrome do túnel do carpo. Rev Bras Ortop 1996; 31:199-202.

5. Gerritsen AA, de Vet HC, Scholten RJ, Bertelsmann FW, de Krom MC, Bouter LM. Splinting vs surgery in the treatment of carpal tunnel syndrome: a randomized controlled trial. JAMA 2002; 88:1245-51.

6. Phalen GS. The carpal-tunnel syndrome. Seventeen years' experience in diagnosis and treatment of six hundred fifty-four hands. J Bone Joint Surg Am 1966; 48:211-28

7. Zumiotti AV, Ohno PE. Tratamento cirúrgico da síndrome do túnel do carpo via endoscópica.Rev Bras Ortop 1994; 29:561-4.

8. Chow JC. Endoscopic release of the carpal ligament: a new technique for carpal tunnel syndrome. Arthroscopy 1989: 5:19-24.

9. Macdermid JC, Richards RS, Roth JH, Ross DC, King GJ. Endoscopic versus open carpal tunnel release: a randomized trial. J Hand Surg Am 2003: 28: 475-80.

10. Fernandes $\mathrm{CH}$, Meirelles LM, Carneiro RS, Faloppa F, Albertoni WM. Tratamento cirúrgico da síndrome do canal do carpo por incisäo palmar e utilizaçäo do instrumento de Paine $\AA$. Rev Bras Ortop 1999; 34:260-70.

11. Haupt WF, Wintzer G, Schop A, Löttgen J, Pawlik G. Long-term results of carpal tunnel decompression. Assessment of 60 cases. J Hand Surg Br 1993; 18: 471-4.

12. Nancollas MP, Peimer CA, Wheeler DR, Sherwin FS. Long-term results of carpal tunnel release. J Hand Surg Br 1995; 20:470-4

13. Phalen GS. The carpal-tunnel syndrome. Clinical evaluation of 598 hands. Clin Orthop. 1972; 83:29-40.

14. Paine KW, Polyzoidis KS. Carpal tunnel syndrome. Decompression using the Paine retinaculotome. J Neurosurg 1983; 59:1031-6.

15. Dellon AL, Mackinnon SE., Brandt KE. The markings of the Semmes-Weinstein nylon monofilaments. J Hand Surg Am 1993; 18:756-7.

16. Szabo RM, Gelberman RH, Dimick MP. Sensibility testing in patients with carpal tunnel syndrome. J Bone Joint Surg Am 1984; 66: 60-4

17. Kellor M., Frost J, Silberberg N, Iversen I, Cummings R. Hand strength and dexterity. Am J Occup Ther 1971; 25:77-83.

18. Mathiowetz V, Kashman N, Volland G, Weber K, Dowe M, Rogers S. Grip and pinch strength: normative data for adults. Arch Phys Med Rehabil 1985; 66:69-74.

19. Young VL, Logan SE, Fernando B, Grasse P, Seaton M, Young AE. Grip strength before and after carpal tunnel decompression. South Med J 1992; 85:897-900.

20. Gellman H, Kan D, Gee V, Kuschner SH, Botte MJ. Analysis of pinch and grip strength after carpal tunnel release. J Hand Surg Am 1989; 14:863-4.

21. Eversmann WW. Entraptament and compression neuropaties. In: Green DP. Operative hand surgery. New York: Churchill Livingstone; 1988. p.1336-66.

22. Viegas SF, Pollard A, Kaminksi K. Carpal arch alteration and related clinical status after endoscopic carpal tunnel release. J Hand Surg Am 1992: 17:1012-16.

23. Wilson KM. Double incision open technique for carpal tunnel release: an alternative to endoscopic release. J Hand Surg Am 1994; 19:907-12.

24. Brown RA, Gelberman RH, Seiler JG 3rd, Abrahamsson SO, Weiland AJ et al. Carpal tunnel release. A prospective, randomized assessment of open and endoscopic methods. J Bone Joint Surg Am 1993; 75:1265-75. 